\title{
Modalité gestuelle et enseignement/ apprentissage du français à l'école primaire
}

\author{
Nabila MAARFIA \& Meriem BOUZIT \\ Département de français, Université Badji Mokhtar - Annaba, Algérie
}

Reçu le 22 avril 2019 | Accepté le 30 juin 2019

\begin{abstract}
RÉSUMÉ. Se préoccuper de l'agir enseignant nécessite forcément l'analyse de son discours, de ses manières de faire, de ses postures... Cet article se penche sur l'aspect non-verbal de cet agir, à savoir la gestuelle enseignante en ce qu'elle contribue à l'accès au sens en langue étrangère. Notre recherche se veut compréhensive et s'inscrit dans une démarche de type ethnographique, recourant principalement à la méthode qualitative et faisant incidemment appel aux données quantitatives afin de mettre en évidence la fréquence et la variation intra-individuelle de la gestualité co-verbale utilisée par l'enseignant à l'école primaire. Les données ont été collectées grâce à l'observation non participante et l'enregistrement filmé de séances de cours d'une même enseignante dans deux classes de niveau institutionnel différent, et les gestes répertoriés ont été classés selon le modèle de Mc Neill pour le type et celui de M. Tellier pour la fonction. Les résultats révèlent que la gestualité de l'enseignante est une construction personnelle, une utilisation variée des différents types de gestes et que ce qui fait la différence entre les deux niveaux c'est à la fois la forme et la récurrence des gestes adoptés. Vu la rareté des travaux en contexte algérien, cette étude est une contribution à l'analyse du geste pédagogique de l'enseignant en classe de FLE en interaction avec ses apprenants afin de rentabiliser cette modalité et d'en tenir compte dans la formation des formateurs.
\end{abstract}

Mots-clés : Agir professoral, geste coverbal, niveau institutionnel, taux gestuel

\begin{abstract}
To be interested about the teacher's action necessarily requires the analysis of his speech, his ways of doing things, his postures... This article examines the non-verbal aspect of this action, namely the teaching gesture in that it contributes to access to meaning in a foreign language. Our approach is comprehensive and ethnographic, using mainly the qualitative method, but also relying, incidentally, on quantitative data in order to highlight the frequency and intra-individual variation of the co-verbal gestuality used by the teacher in primary school. The data was collected by non-participant observation and filmed recording of the sessions of the same teacher in two classes of different institutional levels, and the gestures were classified according to the model of Mc Neill (1992) regarding their type and that of $\mathrm{M}$. Tellier $(2006,2008)$ for their function. The results reveal that the teacher's gestuality is a personal construction, a varied use of the different types of gestures and that what make the difference between the two levels are both the form and the recurrence of the gestures adopted. Given the scarcity of work in the Algerian context, this study is a contribution to analysis of the teacher's pedagogical gesture in the French classroom in interaction with his learners in order to make this modality profitable and to take it into account in the formation of trainers.
\end{abstract}

Keywords: Co-verbal gesture, gestural rate, institutional level, teachers' action

auteur correspondant : maarfianabila@yahoo.fr

Pour citer cet article (Style APA) : Maarfia \& Bouzit. (2019). Modalité gestuelle et enseignement/ apprentissage du français à l'école primaire. Francisola: Revue Indonésienne de la langue et la littérature françaises, 4(1), 12-21. doi: 10.17509/francisola.v4i1.20337. 


\section{INTRODUCTION}

Un intérêt particulier a été réservé, au sein des études sur les pratiques d'enseignement, à l'agir de l'enseignant et plus spécifiquement à l'aspect non verbal de son discours, à cette " manière de faire grâce à laquelle celui qui a élaboré un rapport construit et de "première main" au savoir va assurer la communication de ce savoir, en général au sein d'un dispositif didactique » (G. Sensevy, 2010).

Depuis les années 60-70, beaucoup de chercheurs en sciences du langage et de la communication se sont intéressés à l'étude de la gestuelle allant de la mimique faciale (P. Ekman, 1980), à la perception et la gestion de l'espace (E.T. Hall, 1966), à l'intérêt porté par R. Birdwhistell à la kinésique (1968). A leur suite, A. Kendon (1981), s'est préoccupé du geste en tant qu'unité indépendante qui sert la communication. Mc Neill quant à lui (1992), a étudié le lien geste, parole et pensée en se focalisant sur les gestes des mains.

En 1989, Porcher et Calbris ont souligné l'importance et l'implication du geste pédagogique dans le domaine de la didactique et juste après, en 1990, C. Alin évoquera la notion de "geste professionnel ", qui sera repris en 1998 par A. Jorro qui s'est intéressée, quant à elle, à l'inscription des gestes professionnels dans l'action enseignante, ce que D. Bucheton définira plus tard en 2009 comme $l^{\prime}$ « action pour faire agir et réagir l'autre ». Enfin à partir de 2004, les recherches menées par M. Tellier viendront actualiser le rôle important que joue le geste en cours de langue étrangère le considérant «comme une traduction non verbale des propos de l'enseignant [qui] permet à l'apprenant de saisir le sens des paroles » (2008).
Nous nous interrogeons, dans cet article, à la variation du geste coverbal de l'enseignant en fonction de la situation langagière dans laquelle il est engagé afin de repérer les gestes opérants en début d'enseignement/d'apprentissage d'une langue étrangère, ici le français.

\section{MÉTHODE}

Cette recherche vise à rendre compte des gestes professionnels d'une enseignante de français à l'école primaire en Algérie, selon une perspective descriptive et compréhensive. Nous avons opté pour une approche ethnographique qui est « une façon de se situer face à la réalité sociale et éducative et face à la connaissance de cette réalité, qui par ailleurs est multiple » (Cambra-Giné, 2003, p.15).

Notre corpus a été recueilli suite à une observation non participante et à la transcription des enregistrements filmés de quatre séances de cours, présentées par la même enseignante dans deux classes de l'école primaire Khelfaoui El Hadi située à Essarouel, petite localité de commune d'El Bouni (Wilaya d'Annaba), la première de 3 ème année (première année d'apprentissage du français) et la seconde de 5 ème année (dernière année du cycle primaire). Nous avons choisi l'activité de compréhension orale qui est la première abordée au commencement de chaque séquence et enregistré deux séquences pour chaque projet et chaque classe. Les deux groupes-classe sont constitués comme suit :

- Classe 1- 5ème A.P : 24 apprenants dont 14 filles et 10 garçons âgés de 10 à 12 ans.

- Classe 2- 3ème A.P : 29 apprenants dont 14 filles et 15 garçons âgés de 8 à 9 ans. 
Tableau 1. Récapitulatif des séances

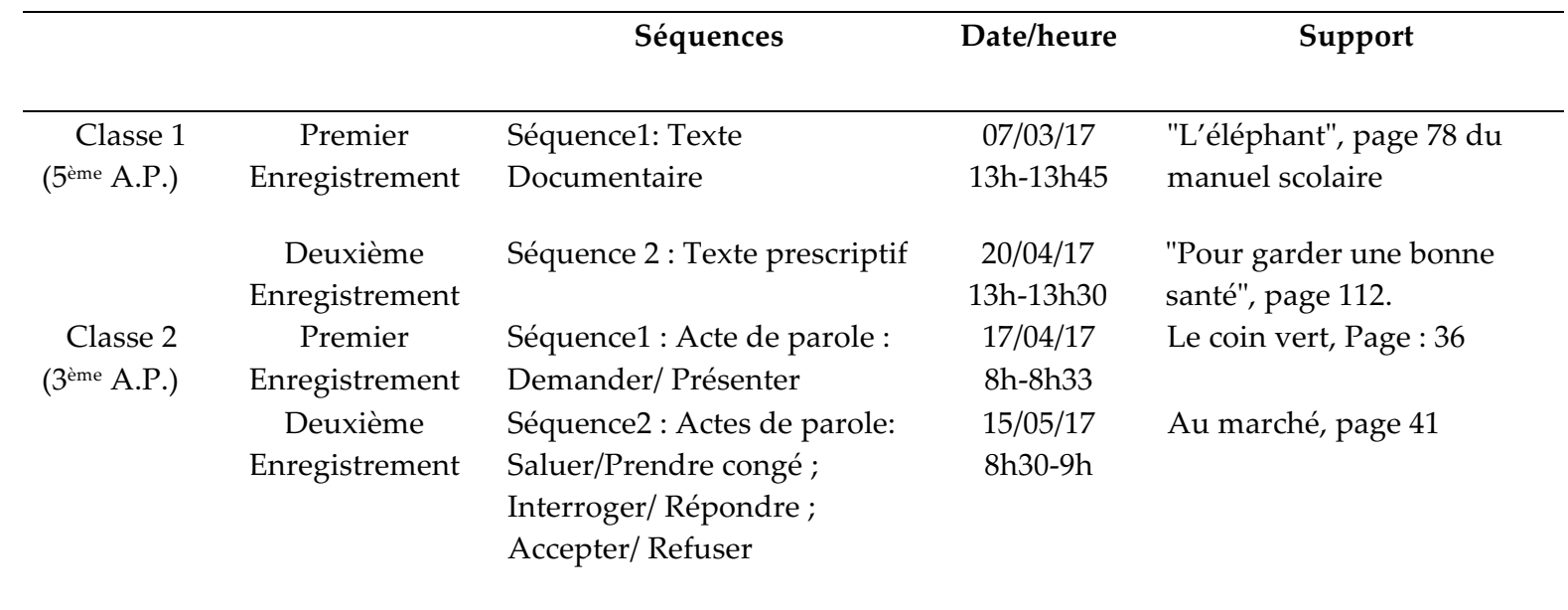

Le choix de cette école primaire, n'est pas anodin mais réfléchi, car notre objectif était d'observer et d'analyser des échanges langagiers d'élèves qui font la connaissance du français au sein de l'établissement uniquement. Ce public, est dans sa grande majorité non exposé à cette langue en dehors de la classe, par opposition à celui du centre ville d'Annaba, qui non seulement y sont souvent exposés mais qui également la pratique souvent chez eux, qui renvoie au lien entre milieu d'appartenance des élèves et les stratégies déployées par l'enseignant pour les faire accéder au sens d'un message en L2 (Maarfia, 2017, p.122) notamment à travers le recours à la gestuelle qui fait l'objet de cette contribution.

Pour ce qui est de l'enseignante, elle a 40 ans et enseigne depuis une quinzaine d'années. Elle est diplômée d'une licence en langue et littérature français mais sans aucune formation initiale, ni pour ce qui est des pratiques enseignantes, ni concernant l'utilisation des gestes pédagogiques.

Pour suivre les interactions orales au sein des deux groupe-classe et constituer notre corpus, nous avons retenu comme outils d'investigation pour le recueil des donnés l'enregistrement vidéo ${ }^{1}$ et l'observation non participante à l'aide d'une grille inspirée de celle de Jean Michel Ducrot, la classe de langue étant considérée « comme une sorte de creuset où se produisent des processus orientés vers l'apprentissage » (Cambra-Giné, 2003, p.97). Notons que nous n'avons pas procédé à de l'annotation gestuelle, mais juste à une transcription audio accompagnée de la description de ce que fait l'enseignante.

\section{RÉSULTATS ET DISCUSSION}

\subsection{Résultats}

L'analyse a porté sur les interactions enseignante/apprenants, en se focalisant sur la gestuelle déployée par l'enseignante avec chacun des deux publics afin d'en observer les variations intra-individuelle (Azoui, 2014). Il en ressort une utilisation de tous les types de geste proposé par David McNeill (1992), à savoir : le déictique (geste de pointage), le battement (geste rythmant la parole, sans contenu sémantique), l'iconique (geste illustrant un concept concret) et le métaphorique (geste illustratif d'un concept abstrait). Ci-dessous un tableau récapitulatif de tous les gestes utilisés par l'enseignante dans les deux groupes-classe2. 


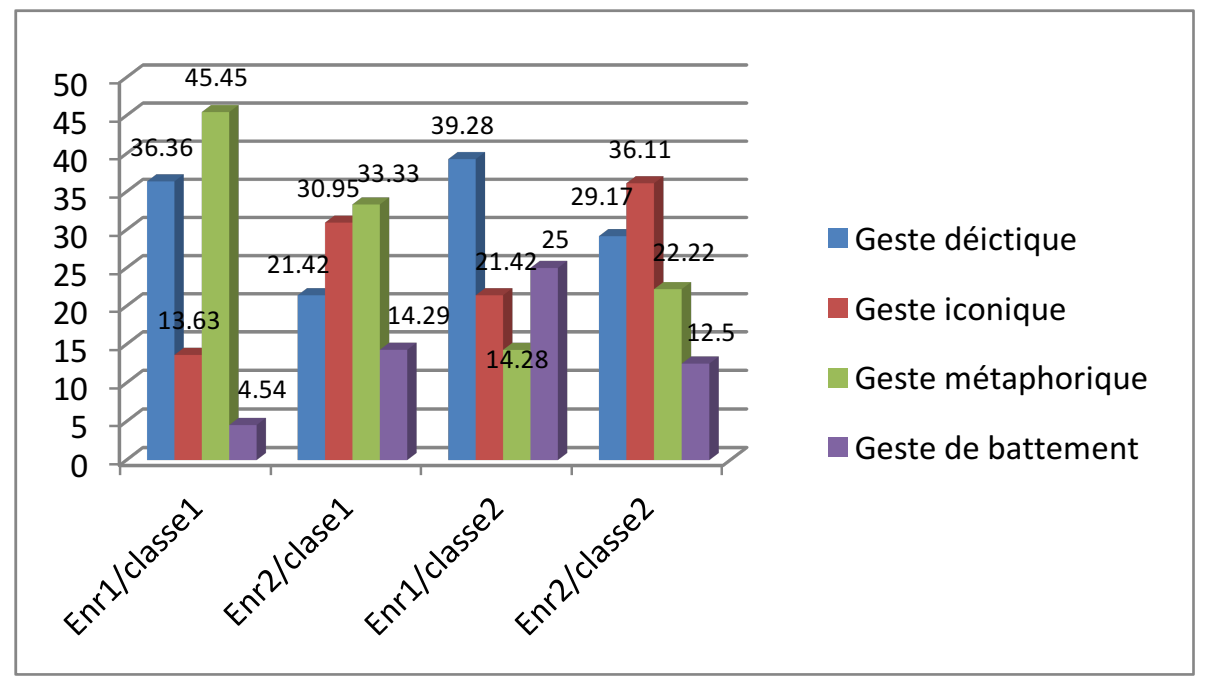

Figure 1. Gestes produits par l'enseignante dans les deux classes pendant les deux enregistrements

\subsubsection{Typologies des gestes employés par l'enseignante}

De cette panoplie de gestes, nous nous sommes intéressées dans cette recherche à ceux coverbaux à visée illustrative et écarté ceux à finalité évaluative, qui feront l'objet d'une autre recherche).

Pour étudier le geste pédagogique et son effet en classe de langue, nous avons procédé en deux temps : d'abord en répertoriant les différents types selon la typologie proposée par McNeill (1992), pour analyser ensuite les fonctions de ces dimensions gestuelles telles que définies par Tellier (2010).

\subsubsection{Les gestes déictiques}

Ces gestes de pointage indiquent un lieu, une personne ou tout autre objet dans l'espace ou le temps. Ils ont donc une fonction principalement référentielle (Azaoui, 2014) et sont plus utilisés chez le groupe-classe de 5 ème AP (désormais G1) à valeur de $39.28 \%$ \& $29.17 \%$, que chez celui de $3^{\text {ème }} \mathrm{AP}$ (désormais G2) qui sont de l'ordre de $36.36 \%$ \& $21.42 \%$. Cette différence renvoie à la nature des supports utilisés : pour le G1, l'enseignante a utilisé un texte lu par ellemême alors que pour le G2, elle a recouru à des saynètes renvoyant à des objets concrets présents dans la classe tels que la fenêtre, les pots de fleurs...). Ajoutons à cela que l'enseignante avait pour objectif principal de faire apprendre aux élèves à montrer et nommer en langue cible des éléments ou objets présents dans la classe en utilisant la monstration « voici ».

Exemple 1:

C1_156_E : d'herbe et de fruits et de racine/vous connaissez ce que c'est les racines, regardez voici un arbre /les petits arbres là «haye »/[voici] la racine/« hame» [ce sont] des racines.

Dans ce tour de parole, l'enseignante revient à l'image qui est sur le tableau et commence son explication (ce que veut dire "racines") en recourant aux déictiques qui appuient le geste de monstration.

Exemple2 :

C2_86_E : voici/ voi :ci /je montre

L'enseignante montre aux élèves le coin vert sur la saynète en utilisant un geste déictique.

\subsubsection{Les gestes iconiques}

Notons que les gestes iconiques ont pour rôle de symboliser un objet concret ou une action. Nous en retrouvons dans les deux classes : $13.63 \%$ \& $30.95 \%$ pour le G1 et $21.42 \%$ \& $36.11 \%$ ) pour le G2. 
Comme nous l'avons expliqué plus haut en se référent à la typologie de McNeill (1992), le geste iconique sert à l'explication sémantique du lexique en langue étrangère. En se référant à la grille d'observation $n^{\circ} 01$, nous remarquons un taux un peu élevé des gestes iconiques de sa part et qui s'explique par sa stratégie d'évitement du recours à la L1 qu'elle ne favorise pas dans le déroulement de la séance. Nous émettons l'hypothèse que ces élèves sont habitués à ces gestes, ce qui les a aidés à accéder au message, entièrement en L2, comme le montre cet exemple où l'enseignante essaie d'expliquer pourquoi l'éléphant mange les racines, tout en évitant de donner la traduction en L1 d'un nouveau mot.

Exemple 3 :

C1_160_E3 : «les racines, pourquoi il mange les racines? je vais vous expliquer/quand il arrache/ il a une main pour arracher? avec quoi il /il arrache ?/avec/avec sa...» (elle recourt ici au geste iconique en dessinant dans l'air avec sa main la trompe de l'éléphant).

\subsubsection{Les gestes métaphoriques}

Pour ce qui est de ce type de gestes métaphoriques, il est plus important chez le G1 $(45.45 \%$ \& $33.33 \%)$ que chez le G2 $(14.28 \%$ \& $22.22 \%)$. Ces résultats ne vont pas dans le sens de ce qu'avancent Tellier, \& Stam (2012) qui prévoient avec les non-natifs un nombre plus important d'iconiques et de déictiques et un nombre moins important de métaphoriques et d'emblèmes dans la condition native. Dans notre cas, cela pourrait s'expliquer par le contenu assez dense, aux référents abstraits, proposé à ces élèves alors qu'ils sont en début d'apprentissage de la L2.

\subsubsection{Geste de battement}

Pour les gestes de battement, nous avons recensé un pourcentage plus élevé chez le G2 (25\% \& $14.29 \%)$ par rapport au G1 $(4.54 \%$ \& $12.5 \%)$. Soulignons que l'enseignante à recours à ce type de geste pour assurer le calme dans la classe et attirer l'attention des apprenants. Nous n'avons dégagé que trois gestes de battement qui servent la correction des erreurs commises par les apprenants.

Exemple 4 :

C1_108_E: voilà !ce texte c'est un texte do:cu:men:taire $\uparrow$

Pour corriger la prononciation des élèves, elle répète le mot documentaire lentement en levant l'index de la main droite vers le haut.

\subsubsection{Raisons de l'utilisation des gestes dans les deux classes}

Durant le déroulement des séances de cours, trois fonctions peuvent être simultanément remplies par l'enseignant : celle d'informateur, d'animateur et d'évaluateur (Dabène, 1984). De ce fait, ce dernier mobilise de multiples moyens qui sont à la fois verbaux, non verbaux et paraverbaux : explication d'un mot, allongement vocalique pour accentuer un énonce linguistique et recours au geste pour illustrer une idée ou un mot (Tellier, 2006). Cette multimodalité de l'agir professoral (prosodique, mémo-gestuelle et verbale) est considérée comme un élément essentiel dans le processus d'apprentissage d'une langue étrangère (Azaoui, 2014).

\subsubsection{Les déictiques}

Dans les deux classes observées, nous avons remarqué l'abondance des déictiques, notamment pour ce qui est de la classe de 3ème A.P. Ils sont produits tout au long du déroulement $\mathrm{du}$ cours pour les raisons suivantes :

\section{a) Pour expliquer}

Exemple 5:

C1_135_E : Asie, l'éléphant habite ou bien vit en Afrique et en A:sie/ bien// regardez, il vit ici l'éléphant ah «haye l'Afrique» (c'est ici l'Afrique). Il habite dans la savane africaine, Le Sénégal Ah! ici, on trouve l'éléphant et aussi on le trouve en A:sie, d'accord? L'éléphant asiatique est moins grand que l'éléphant africain. L'éléphant africain est plus grand (en utilisant un geste «petit et grand, même dans 
la couleur ils diffèrent. Le mois d'avril on aura un texte sur l'éléphant et vous allez voir la différence entre l'éléphant d'Afrique et l'éléphant d'Asie/ les oreilles, elles sont plus petites, Ah! d'accord? donc, l'éléphant habite en Afrique et en Asie. qu'est-ce qu'il mange?

Après l'évaluation positive de la réponse apprenante, l'enseignante va montrer aux élèves où vit l'éléphant. L'enseignante se dirige vers la carte géographique collée sur le mur et montre où est la position de l'Afrique. C'est le recours au déictique «ici » qui appuie le geste de monstration sur la carte.

\section{b) Pour solliciter et donner la parole aux apprenants}

Par l'intermédiaire du geste, l'enseignante désigne un élève. Cela peut être expliqué par le fait que l'enseignante a oublié le nom de l'élève ou bien ce dernier se trouve loin d'elle (au fond de la classe), comme dans l'exemple suivant, à l'aide du geste, l'enseignante demande aux apprenants de prendre la parole pour répéter le titre du texte.

\section{Exemple 6 :}

C2_31_E: consonne (rire) santé/encore! haya on répète (elle désigne du doigt l'élève F).

C2_32_F : le titre de texte pour

C2_33_E : pour garder

C2_34_F : pour garder/// en en $\varnothing$

C2_35_E : une bonne $\uparrow$

C2_36_F : une bonne santi

C2_37_E : santé $\uparrow$

C2_38_F: santé

C2_39_E: bien /(elle désigne du doigt l'élève G.41)

C2_40_As : madame! madame !

C2_41_G: le titre de texte pour garder une bonne santé

C2_42_F : le titre texte (désignée du doigt)

C2_43_E : du

C2_44_F : de texte pour garder une bonne santé
C2_45_F: le titre de texte pour garder une bonne/ bonne santé (désignée du doigt)

\section{c) Pour illustrer}

Dans le but de guider les apprenants et les laisser parler, l'enseignante utilise le geste déictique.

Exemple 7 :

C1_225_F : Madame quatre dents.

C1_226_E: « aha » (non) c'est pas des dents ça

C1_227_G: madame défense

C1_228_E: très bien (évaluation positive), des/il a deux défenses.

Dans cet exemple, l'enseignante a signalé une erreur en employant le «non » réfutatif, puis, désigné les défenses dessinées sur l'image. Le geste déictique précise le «ça » qui désigne les défenses. Il a donc une fonction illustrative.

\subsubsection{Les gestes iconiques}

Un geste iconique représente un objet concret, donc nous le trouvons beaucoup dans l'explication (explication sémantique d'un mot). Ils sont utiliser pour :

\section{e) Enumérer}

Exemple 8 :

C1_356_F : il avale 200 kilos de fruit

C1_357_E: de

C1_358_F : d'herbe, de racine/de racine par jour

Dans cet exemple, l'enseignante pour aider l'élève à terminer sa réponse, recourt au geste iconique : elle énumère avec la main et l'élève dans le tour de parole suivant termine la réponse.

Exemple 9 :

C3_7_F : je vois la rose

C3_8_E : la $\uparrow$ rose ?

C3_9_F : XXX

C3_10_E : ah les:: roses / ah

Pour corriger la réponse de l'élève, l'enseignante revient sur l'image et compte avec les doigts les roses dessinées en pointant le pouce, l'index et le majeur pour montrer $\mathrm{qu}^{\prime}$ il y a trois roses. 


\section{b) Expliquer un nouveau lexique}

Exemple 10:

C1_160_E: les racines, pourquoi il mange les racines? je vais vous expliquer quand il arrache il a une main pour arracher ?avec quoi il/il arrache ?avec/ avec sa : (geste : pour dessiner la trompe de l'éléphant, elle part de son nez en allongeant sa main)

C1_161_G : Madame avec sa trompe C1_162_E : très bien, avec sa trompe, c'est comme une main, c'est comme une main.il fait comme ça et "hope » il avale je vais vous dire une chose les racines c'est plein d'eau, il y a de l'eau dedans. ah! les racine (elle montre les racines sur l'image)

Dans cet extrait, l'enseignante veut expliquer pourquoi l'éléphant mange les racines. Pour dire qu'il arrache les plantes avec sa trompe, elle l'a dessinée dans l'air. Ce geste accompagne une demande d'achèvement interactif pour solliciter les apprenants à dire le mot produit par le geste.

c) Pour expliquer les mots importants dans le texte

Exemple 11:

C2_17_E: un texte qui donne des conseils/ alors j'écoute pour garder une bonne santé/ comme nous sommes tristes quand nous sommes malades! comme c'est pénible! c'est pourquoi nous voulons tous rester en bonne santé./pour maintenir notre corps en bon état, il nous faut beaucoup d'air pur à respirer. Les enfants de la compagne ou du bord de la mer le trouvent chez eux. Pour les enfants des villes, il a fallu créer des colonies de vacances. Ils peuvent donc respirer l'air pur./ pour rester en bonne santé, il nous faut encore beaucoup d'eau pure à boire et des aliments bien choisis: des laitages, des protéines, des céréales./ les enfants ont besoin de plus de repos que les grandes personnes. En effet leur corps doit non seulement se réparer mais aussi grandir. c'est le sommeil qui est le meilleur repos. d'accord ?/ on a bien écouté/j'écoute une deuxième fois vous gardez le plus important//

En lisant le texte, l'enseignante essaie d'expliquer les mots-clés dans le texte pour faciliter la compréhension et afin que les élèves les mémorisent et les réemploient dans la production orale. Elle dessine les larmes sur ses joues, elle respire de l'air, etc.

d) Pour montrer le terme manquant dans la réponse de l'élève

Exemple 12:

C1_400_G: et de racine/ l'éléphant vit en

C1_401_E : en (avec sa main) pour dire troupeau

C1_402_G: en troupeau/ l'éléphant vit toujours /à coté $/ \mathrm{d}^{\prime}$ :un : point /d'eau

L'enseignante a demandé à l'élève de lire ce qui est écrit sur le tableau. Pour l'aider à terminer sa phrase, elle recourt au geste iconique (elle ouvre les mains et rapproche les deux bras) pour dire troupeau ou famille. Ici le geste iconique aide l'enseignante dans l'évaluation de la réponse de l'élève.

\subsubsection{Les métaphoriques}

Contrairement aux gestes iconiques, les gestes métaphoriques représentent des concepts abstraits. Ces gestes sont produits par l'enseignante pour :

a) Pour poser la question

Exemple 13:

C3_1_E: regardez la vignette les enfants/ qu' est ce que je vois?

C3_2_F : je vois les élèves $\downarrow$

L'enseignante pose la question. Pour ne pas la répéter une seconde fois, elle a recourt au geste interrogatif (elle tourne sa main dans l'air en traçant un demi-cercle) suivi d'un geste iconique (l'index placé en dessous de l'œil). Ici ce geste sert à expliquer le sens de la question et à pousser les élèves à répondre. L'apprenante interprète ces gestes et répond par « je vois les élèves ».

\section{b) Pour inciter les élèves à parler encore (à donner d'autres exemples)}

Exemple 14 :

C2_229_E : Hanine euh Yakine 
C2_230_F(hanine): pour garder une euh une bonne santé

C2_231_E : enlève ta feuille $\mathrm{mm}$

C2_232_F(Hanine) : faut

C2_233_E : il faut

C2_234_F(Hanine) : il faut faire du sport

C2_235_F(Yakine): il faut respirer l'air pur

C2_236_E: bien «haya» changez/ changez

C2_237_As: madame madame

C2_238_E: Soundes avec euh Hadjer

Dans cet extrait, l'enseignante demande aux élèves de donner des conseils pour avoir une bonne santé. Elle a choisi deux élèves pour le jeu de rôle, Hanine et Yaqine. Son évaluation positive vient à la fin suivie d'un geste métaphorique (les mains tournées dans l'air plusieurs fois pour dire « changer ») afin d'inciter les autres élèves qu'elle a choisi, Soundes et Hadjer, à donner de nouveaux exemples.

Exemple 15 :

C2_255_F : il faut faire sport

C2_256_E: du

C2_257_F : du sport

C2_258_F : il feu manger

C2_259_E : il faut $\uparrow$

C2_260_F :il faut manger de fruit et e légume

C2_261_E: « hein » c'est tout! faire du sport et manger des légumes c'est tout $\uparrow$

Dans cet exemple, nous retrouvons le même objectif: donner d'autres exemples, mais en produisant un autre geste métaphorique en mettant la paume droite dans la paume gauche puis la gauche dans la droite pour signifier «c'est tout?», car les élèves reprenaient les mêmes exemples : faire du sport, manger les légumes et les fruits).

\subsubsection{Les gestes de battement}

Nous avons remarqué que

l'enseignante recourt à ce type de geste pour ramener le calme en classe en employant l'interjection «chut!». Mais les battements ont aussi une fonction évaluative.

Exemple 16:
C1_16_G: je vois en/en l'etélé l'eléphant.

C1_17_E : Ah! tu as vu à la té:lé:vi:sion $\uparrow$

Dans cet exemple, le contenu de la réponse apprenante est correct mais la forme est erronée. L'enseignante attire l'attention de l'élève en élevant sa voix et en découpant le mot en syllabe accompagné d'un mouvement saccadé du doigt pour l'accentuer.

\subsection{Discussion}

L'analyse de notre corpus révèle que les gestes produits par l'enseignante ont différentes fonctions: d'information, d'animation et d'évaluation (Tellier, 2006, 2008). Ces trois fonctions se réfèrent à celles proposées par Dabène (1984) pour la parole de l'enseignant de langue, ce qui renvoie au lien entre geste et parole.

Pour la première catégorie (geste d'information), nous avons vu que l'enseignante fait appel aux gestes déictique, iconique et métaphorique dans une visée explicative. Ces derniers contribuent à la fois à faire accéder au sens en langue étrangère et à provoquer la parole apprenante.

Pour la deuxième catégorie (les gestes d'animation), nous les trouvons dans les interactions enseignant/apprenant(s), dans le dialogue interrogatif: lorsque l'enseignante pose des questions, les répète, sollicite les apprenants et leur donne la parole, en utilisant beaucoup de déictiques.

Enfin, il est à noter que l'enseignante déploie différentes stratégies: verbal, paraverbal et non-verbal, qui sont destinées à la coopération et l'étayage en vue de résoudre un problème de compréhension orale chez l'élève et afin de l'aider à prendre la parole à son tour et à s'exprimer en LE.

\section{CONCLUSION}

La présence des gestes pédagogiques représente un outil pour l'enseignant et une aide pour l'apprenant. L'enseignant fait appel à la gestuelle tout au long du déroulement de la séance pour expliquer, pour faciliter la compréhension aux élèves, 
pour évaluer leurs réponses tout en évitant le recours à la L1.

Nous rejoignons M. Tellier (2014, p.185), quand elle affirme que « la gestuelle permet à la fois la focalisation des apprenants sur la parole de l'enseignant et favorise une meilleure compréhension », du fait que l'apprenant est doublement servi, à travers la modalité auditive et celle visuelle.

Nous recommandons le recours à la gestuelle, qui peut à notre sens faire l'objet d'un enseignement conscient car l'enseignant (notamment le novice) «est parfois focalisé sur les éléments linguistiques à transmettre au point de se priver de l'utilisation du geste comme support» (Tellier, p. 2017).

Pourquoi se priver du recours aux gestes, support plus facile à produire spontanément et naturellement en interaction pédagogique, qui offre une aide considérable du fait que le discours de l'enseignant gagne à être illustré. C'est cette association d'un geste renforçant la parole dans une approche multimodale qui permet à l'élève une réelle construction du sens (Azaoui, 2014).

\section{REMERCIEMENTS}

Nous tenons à remercier tous les organismes et/ou toutes les personnes qui ont apporté une contribution significative à la rédaction et/ou à l'amélioration de l'article.

\section{RÉFÉRENCES}

Alin, C. (1990). Je, je(ux) et en-je(ux) d'enonciation (les strategies de discours d'enseignants-formateurs dans des situations de communication professionnelle), Thèse de doctorat, Université de Caen, sous la direction de Jean Guglielmi.

Azaoui, B. (2014). Multimodalité des signes et enjeux énonciatifs en classe de FL1/ FLS. Dans M. Tellier et L. Cadet (Eds), Le corps et la voix de l'enseignant: théorie et pratique, Paris, Maison des langues, 115-126.

Birdwhistell, R.L. (1971). Kinesic and Context: Essays on Body-Motion Communication. Philadelphia. Philadelphia, University of Pennsylvania Press.
Bucheton, D. (Dir.). (2009). L'agir enseignant: des gestes professionnels ajustés. Toulouse, Octarès.

Calbris, G. et Porcher, L. (1989). Geste et communication. Paris, Hatier-Crédif.

Cambra-Giné, M. (2003). Une approche ethnographique de la classe de langue étrangère. Paris, Didier. Collection Langues et Apprentissage des Langues.

Dabène, L. (1984). Pour une taxinomie des opérations métacommunicatives en classe de langue étrangère. Etudes de linguistique appliquée, $\mathrm{n}^{\circ}$ 55, 39-46.

Ekman, P. (1980). L'expression des émotions. La Recherche 11, 117, 1408-1415.

Hall, E.T. (1966). The hidden dimension. Garden City, N.Y.: Doubleday. Traduction française de 1971, La dimension cachée, Paris, Editions du Seuil, Coll. « Points »,

Jorro, A. 1998. L'inscription des gestes professionnels dans l'action. Revue en question, 1998, 19, halshs-0011234

Kendon, A. (1980). Gesture and speech: two aspects of the process of utterance. Dans M. R. Key (éd.), Nonverbal Communication and Language, La Haye, Mouton, 207-227.

Kendon, A. (1981). Nonverbal communication, interaction and gesture. The Hague, Mouton.

Maarfia, N. (2017). Agir professoral et étayage interrogatif en début d'apprentissage du français. Etudes en Didactique des Langues (EDL), 29, 117128. Université Toulouse III. arlap.hypotheses.org/10810.

McNeill, D. (1992). Hand and mind: what gestures reveal about thought. Chicago, Chicago University Press, 104-133.

Sensevy, G. (2010). Notes sur la notion de geste d'enseignement. Travail et formation en éducation, 5. Repéré à : http:// tfe.revues.org/1038

Tellier, M. (2004). L'impact du geste dans la compréhension d'une langue étrangère. In Faut-il parler pour apprendre? Dialogues, verbalisation et apprentissages en situation de travail à 
l'école : acquis et questions vives, IUFM Nord Pas-de-Calais, Arras, 24-26 Mars 2004. Sur Cédérom.

Tellier, M. (2006). L'impact du geste pédagogique sur l'enseignement/apprentissage des langues étrangères : Etude sur des enfants de 5 ans. Thèse de doctorat en Science du langage, Université Paris 7, Paris. Repéré à : https://halshs.archivesouvertes.fr/tel-00371041/document

Tellier, M. (2008). Dire avec des gestes. Le Français dans le monde. Recherches et applications, Paris : Le Français dans le monde, 40-50.

Tellier, M. (2010). Faire un geste pour l'apprentissage: Le geste pédagogique dans l'enseignement précoce : Impact sur le développement de la langue maternelle. Dans C. Corblin, L'enseignement des langues vivantes étrangères à l'école, Paris, L'Harmattan, 31-54.

Tellier, M. et Stam, G. (2012). Stratégies verbales et gestuelles dans l'explication lexicale d'un verbe d'action. In V. Rivière (éd.), Spécificités et diversité des interactions didactique, 357-374. Paris: Riveneuve Éditions.

Tellier, M., Michel, L. et Wolff, L. (2014). "Variations inter et intra - individuelles de la gestuelle chez l'enseignante de maternelle in M. Tellier et L. Cadet, Le corps et la voix de l'enseignant: théorie et pratique. Paris, Editions Maison des Langues, 127-140.

\section{NOTES}

1 Seule l'enseignante a été filmée, avec son consentement.

2 Notons que "classe 1" renvoie à celle de la 5 ème A.P et "classe 2 " désigne la 3ème A.P.

3 E 1, 2, 3 : enseignante, C1: classe 1, C2: classe 2, As : plusieurs apprenants, G : apprenant garçon non identifié, $F$ : Apprenant fille, avec le prénom entre (), $\varsigma$ : Son bruissé, $\uparrow$ : Intonation montante, ( ) : traduction en français, $(())$ : commentaire de l'enquêtrice, : : Allongement de la syllabe, / : Pause courte, // : Pause moyenne. 